IdeAs

Idées d'Amériques

$3 \mid 2012$

L'alimentation dans les Amériques au prisme des sciences sociales

\title{
Réflexions sur l'alimentation (Festival de films documentaires AlimenTERRE)
}

Alexandra FAROUX

\section{(2) OpenEdition}

Journals

Édition électronique

URL : https://journals.openedition.org/ideas/540

DOI : $10.4000 /$ ideas. 540

ISSN : 1950-5701

Éditeur

Institut des Amériques

Référence électronique

Alexandra FAROUX, "Réflexions sur l'alimentation (Festival de films documentaires AlimenTERRE) », IdeAs [En ligne], 3 | 2012, mis en ligne le 13 décembre 2012, consulté le 19 octobre 2022. URL : http:// journals.openedition.org/ideas/540; DOI : https://doi.org/10.4000/ideas.540

Ce document a été généré automatiquement le 19 octobre 2022.

\section{(2) $(\mathbb{Q} \Theta \Theta$}

Creative Commons - Attribution - Pas d'Utilisation Commerciale - Pas de Modification 4.0 International - CC BY-NC-ND 4.0

https://creativecommons.org/licenses/by-nc-nd/4.0/ 


\title{
Réflexions sur l'alimentation (Festival de films documentaires AlimenTERRE)
}

\author{
Alexandra FAROUX
}

1 L'industrialisation de la société a modifié nos modes de consommation, et nos pratiques alimentaires s'en sont trouvées bouleversées. L'incroyable diffusion au niveau mondial de la restauration rapide, les prises de repas "sur le pouce", et toutes les autres nouvelles formes d'alimentation «à l'occidentale» symbolisant la modernité, ont participé à la transformation alimentaire traditionnelle.

2 Or, aujourd'hui on assiste à une prise de conscience croissante des individus sur la revalorisation de l'alimentation, ce qui tend à redonner ses vertus, aussi bien physiologiques que culturelles. On voit émerger de cette prise de conscience un intérêt croissant pour l'agriculture, les meilleurs modes de production pour l'homme et l'environnement, et l'importance de refaire le lien entre production et consommation. La question de fond est de comprendre pour quelles raisons l'homme n'utilise-t-il pas son intelligence pour harmoniser ses besoins avec celles du monde qui le nourrit. Ce qui nous renvoie aux pratiques ancestrales et aux modes d'alimentation plus respectueux envers la nature.

3 De nombreuses initiatives pour favoriser l'alimentation de proximité témoignent d'une volonté de parvenir à une alimentation de qualité. Ce terme nous fait penser à la fois à des conditions de production plus respectueuses vis-à-vis de l'environnement, dont la culture limite les intrants chimiques, une alimentation dite «de proximité » permettant aux producteurs locaux de vivre dignement de leur production et assurant la promotion du lien entre le producteur et le consommateur, ainsi que d'une alimentation respectant la saisonnalité des produits.

4 L'émergence des Associations pour le Maintien d'une Agriculture Paysanne (AMAP), à l'instar du modèle teiki japonais, rencontre un franc succès depuis une dizaine d'années. D'autres initiatives fleurissent aujourd'hui telles que les jardins partagés qui 
impliquent un investissement financier moindre, ou plus largement l'agriculture urbaine permettant l'accès des régions urbaines à un potager.

5 Pour toutes les générations, la promotion d'une agriculture durable est aujourd'hui une préoccupation majeure. Cependant la sensibilisation et la promotion de ces initiatives doivent être maintenues et soutenues dans la mesure où il existe une prise de conscience collective. Pour autant, la généralisation des pratiques n'est pas acquise et la reconnaissance des pouvoirs publics reste encore mitigée.

6 Les crises alimentaires mondiales qui se sont produites par le passé, les catastrophes naturelles comme celle qui s'est produite en Amérique en octobre dernier avec l'ouragan Sandy, rendant impossible les approvisionnements en nourriture à la population cubaine et de République Dominicaine par exemple, montrent l'urgence de donner aux Etats les moyens d'assurer leur sécurité alimentaire en produisant localement et en ne dépendant pas de l'extérieur.

7 À l'initiative de Minga, Nature \& Progrès, et à l'occasion de l'élection présidentielle, de nombreuses organisations et personnalités se sont mobilisées pour «faire de l'alimentation une priorité et un projet de haute nécessité démocratique $»^{1}$. Les différentes campagnes menées dans cette dynamique (Alimentons 2012, Alimentons la Démocratie, Alimentons-Nous!), avaient toutes ce même objectif de faire de l'alimentation un véritable sujet de société qu'il est important de mettre au cœur du débat, et la nécessité de nourrir les idées autour du sujet.

8 Le Comité français de solidarité internationale (CFSI) organise chaque année depuis 2007 (du 15 octobre au 30 novembre) le festival de films documentaires AlimenTERRE ${ }^{2}$. Ce festival est le temps fort de la campagne d'éducation au développement portant le même nom et qui a pour principal objectif de mettre en lumière les enjeux alimentaires et agricoles globaux en rendant ces problématiques accessibles à tous. A travers des projections-débats autour de différents documentaires, courts, moyens et longs métrages, cette manifestation permet de rendre visible ces questions, de refaire le lien entre l'alimentation et l'agriculture et de remettre ces thématiques au cœur du débat contemporain.

Cette année parmi les films sélectionnés, La era del buen vivir (2011) de Aline Dehasse, Jeroen Verhoeven et Loïc Dehasse, met en évidence le poids de 500 ans de colonisation et les effets d'une guerre civile sanglante, et la manière dont les mayas, qui habitent aujourd'hui au Guatemala, font face à la globalisation de la société de consommation ; mais aussi comment ils ont des difficultés d'accès à la terre, au pillage des ressources naturelles et aux impacts du réchauffement climatique. Bien loin des conceptions imposées par l'occident, ils construisent des solutions pour demain mettant en pratique leur propre définition du développement.

Le film La face cachée des agrocarburants (2011) d'An Baccaert, Nino Muñoz et Cristano Navarro met en lumière une autre problématique menaçant la souveraineté alimentaire mondiale et montrant la manière dont les indiens Guaranis-Kaiowa de la région Mato Grosso Do Sul à l'ouest du Brésil ont perdu 90\% de leur territoire depuis le début du $20^{\mathrm{e}}$ siècle. Le cœur du problème est l'expansion, après celle du bétail et du soja, des cultures de canne à sucre pour produire de l'éthanol. Le résultat n'est plus surprenant : la faim. " Un comble pour ce peuple dont la variété des aliments faisait autrefois l'admiration. Aujourd'hui confinés dans des réserves ils se battent pour retrouver leurs droits et leurs terres ${» 0^{3}}^{3}$ 
11 La réalisatrice Marie-Monique Robin, à travers son tour du monde des initiatives alternatives de production agricole, prouve dans son dernier documentaire Les moissons du futur (2012), dans une optique de durabilité, que l'avenir de l'agriculture doit être raisonné. On a tendance à croire que l'agro-écologie est un modèle qui ne peut être rentable que sur de petites surfaces. Or, son documentaire rend compte qu'en Allemagne comme aux Etats-Unis de grands propriétaires agricoles admettent qu'une reconversion à un mode de production plus respectueux de l'environnement n'entraîne pas pour autant une perte de rendements des cultures. Ce documentaire montre un renouveau des pratiques agricoles ancestrales comme solution pour l'agriculture de demain et comme alternative à la monoculture intensive qui requiert un usage intensif de produits chimiques. "Pluriculture au Mexique ou au Japon, agroforesterie au Malawi, méthode du push-pull au Kenya - des plantes repoussent herbes et insectes nuisibles au maiis, quand d'autres les attirent... Partout, des petits producteurs à la conscience aiguë témoignent des bénéfices vertueux de leur (re)conversion à l'agriculture biologique. Le sens commun a plus ou moins imposé l'idée que l'agroécologie est une utopie et qu'elle seule ne pourrait suffire à nourrir le monde. Mais si la solution se trouvait dans la monoculture, l'utilisation massive d'engrais chimiques couramment utilisés dans l'agriculture dite « conventionnelle », comment se fait-il que ce modèle ne satisfasse pas les besoins en nourriture de la population et que près d'un milliard de personnes continue à souffrir de la faim ?

Dans moins de quarante ans nous serons 2 milliards d'être humains supplémentaires sur la surface de la terre. Faut-il continuer à favoriser la production intensive à grande échelle, alors qu'on a pris conscience de toutes ses conséquences pour l'environnement et pour la santé ? On s'aperçoit que le problème n'est finalement pas tellement lié à la quantité, car on mesure le gaspillage alimentaire et d'autres pertes liées au mode de production dominant à 20 kilos par an et par personne en France (selon les chiffres du Ministère de l'Agriculture). Certes, il faudra produire davantage, mais ne faudra-t-il pas surtout produire mieux et donner aux pays les capacités de nourrir leur population et qu'ils atteignent la souveraineté alimentaire? Au risque de gêner les intérêts de grandes multinationales de l'agroalimentaire, de mettre à jour les contradictions de la politique agricole commune de l'Union Européenne et de bien d'autres acteurs qui profitent du système agricole actuel. Sans l'ombre d'un doute.

\section{NOTES}

1. « Alimentons 2012 », Minga, 2012. http://www.minga.net/spip.php?rubrique123

2. Alimenterre. festival de films documentaires. http://www.festival-alimenterre.org/

3. «La face cachée des agrocarburants ", Alimenterre. festival de films documentaires, 2012. http://

www.festival-alimenterre.org/film/face-cachee-agrocarburants 


\section{AUTEUR}

\section{ALEXANDRA FAROUX}

Diplômée du Master Pro Relations Interculturelles et Coopération Internationale (RICI) à

l'Université Charles de Gaulle/Lille 3. Email : alexandrapointfarouxatgmailpointcom. 